\title{
Roger Alperin
}

\section{Benson Farb and Peter Shalen}

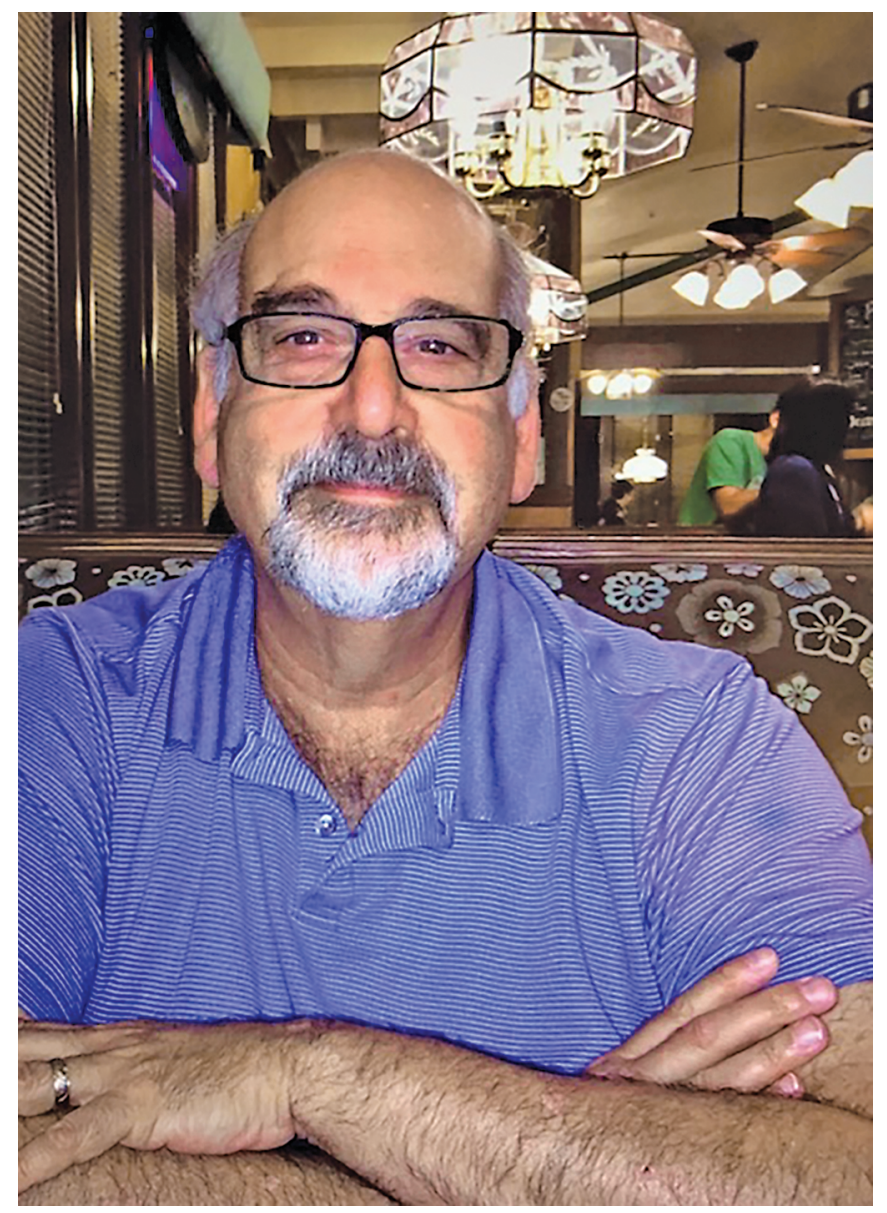

Figure 1. Roger Alperin during a trip to Canada, 2018.

Benson Farb is professor of mathematics at the University of Chicago. His email address is bensonfarb@gmai1. com.

Peter B. Shalen is professor emeritus in the Department of Mathematics, Statistics, and Computer Science at the University of Illinois at Chicago. His email address is petershalen@gmai1.com.

For permission to reprint this article, please contact: reprint-permission@ams.org.

DOI: https://doi.org/10.1090/noti2189
Roger Alperin died peacefully on November 21, 2019, at his home in Carlsbad, California. He was 72. Alperin was a gifted mathematician who worked in a number of areas of mathematics with an algebraic flavor. One of the things that made Roger special was that he seemed oblivious to trends, choosing instead to work on projects that appealed to his excellent mathematical taste. In doing this he made a number of surprising discoveries, several of which had significant impact.

Alperin completed his PhD at Rice in 1973 under the direction of Steve Gersten. In 1978, after holding temporary positions at Brown, Haverford, and Washington University, he joined the faculty of the University of Oklahoma, where he was later promoted to the rank of Professor. It was typical of his disregard for convention that when, in 1987, he had personal reasons for moving to California, he simply resigned his position at Oklahoma without having a replacement position. He quickly obtained an appointment at San Jose State, where he remained until his retirement in 2015 .

While Alperin's research career began conventionally enough, focusing on algebraic K-theory and on the closely related topic of homology of arithmetic groups, his work would come to encompass a remarkable range of topics. The diversity and wonderful quirkiness of Alperin's work can be seen in some of the titles of his papers, such as: "A quartic surface of integer hexahedra"; "Some representations of groups of automorphisms of a free group"; "SL $\mathrm{SL}_{2}(\mathbf{Z}[(1+\sqrt{5}) / 2])$ "; " $p$-adic binomial coefficients mod $p$ "; "Origami flat folded states and their moduli"; "Reflections on Poncelet's pencil"; "Undistorted solvable linear groups"; and "Nonvanishing of algebraic entropy for geometrically finite groups of isometries of Hadamard manifolds." Here we shall only describe a few of his contributions that are particularly close to our own interests.

In the early 1980s, Alperin recognized a link between two theories that grew out of quite different mathematical cultures: the work of I. Chiswell and R. Lyndon on length functions in groups, introduced in the setting of 
combinatorial group theory; and the work of J. Tits on treelike objects that occur as 1-dimensional affine buildings for algebraic groups over fields with indiscrete rank 1 valuations. Alperin's paper on this connection was one of the inspirations for the explosion of the use of $\mathbf{R}$-trees during the following decade, from the work of Morgan-Shalen on degeneration of hyperbolic structures, to the use of actions on $\mathbf{R}$-trees as a basic tool in geometric group theory (e.g., in work by Paulin, Bestvina, Rips, Sela, and others). Alperin's own work with Bass on $\Lambda$-trees was part of this development.

Another surprise came when Alperin realized that some of Shalen's ideas on 3-manifolds could be used in a completely different context: the study of the virtual cohomological dimension of finitely generated groups of invertible complex matrices. Alperin and Shalen found the exact condition characterizing those finitely generated matrix groups whose virtual cohomological dimension is finite; in particular this is the case for any finitely generated subgroup of the unitary group $U(n)$. In part because of the surprising simplicity of the statement, their main result and the methods developed in proving it have found applications to work as diverse as Bucher, Connell, and Lafont's work on the vanishing of simplicial volume for certain affine manifolds, and Guentner, Higson, and Weinberger's work on the Novikov conjecture for linear groups.

Roger had an innate kindness that made him liked by everyone who met him. One of us was lucky enough, as an undergraduate, to meet Roger (who was already an established mathematician) at a summer workshop. It took this undergraduate by surprise that Professor Alperin treated him in exactly the same way as he was treating senior colleagues. It was clear that this was not the result of any effort on Roger's part to help a younger mathematician; it simply never seemed to occur to Roger to treat people differently according to differences in their status.

We always had a smile on our face when we were around Roger. This was in part because of his self-effacing wit and his habit of listening carefully to others; and in part it was because he always had a smile on his face. We will miss him.

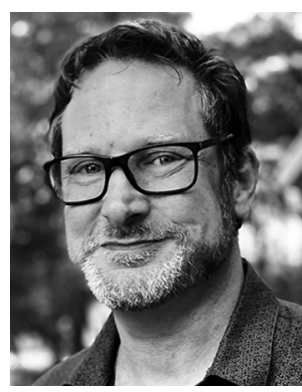

Benson Farb

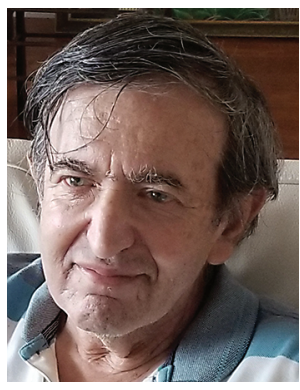

Peter Shalen

\section{Credits}

Figure 1 is courtesy of Gaye Lending Alperin.

Photo of Benson Farb is courtesy of Jessica Wynne.

Photo of Peter Shalen is courtesy of Catherine Shalen.
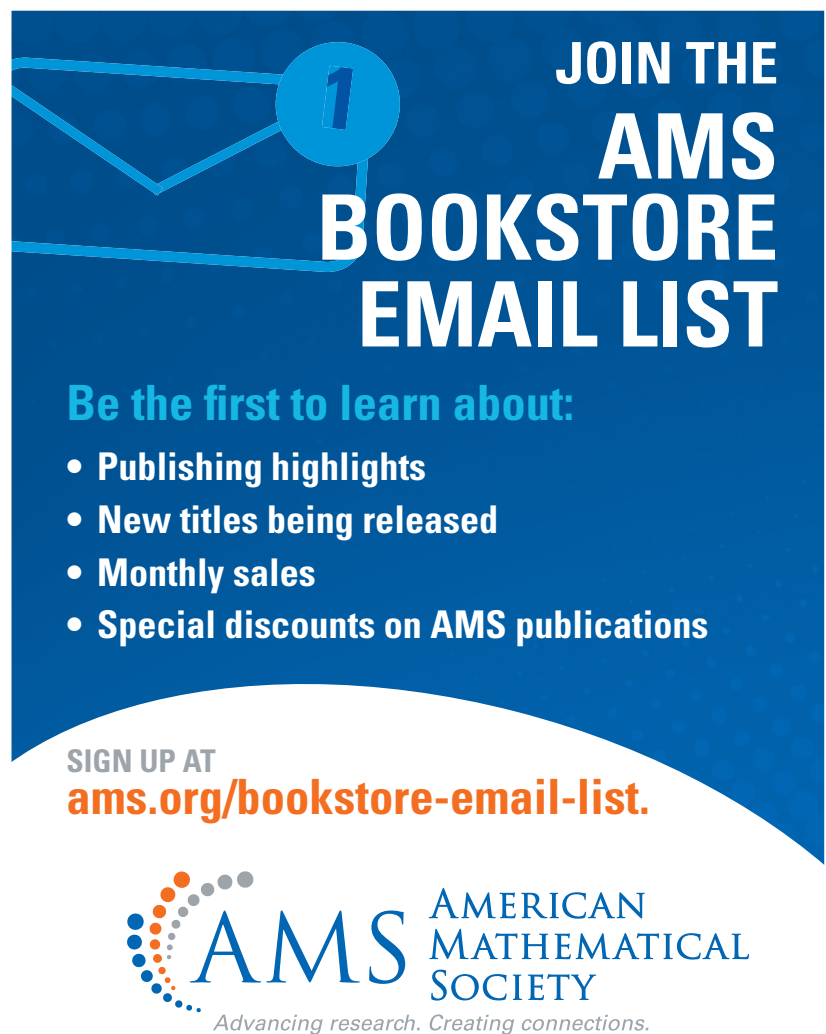